Pesq. Vet. Bras. 35(2):115-118, fevereiro 2015 DOI: 10.1590/S0100-736X2015000200003

\title{
Intoxicação experimental pelos frutos de uva-Japão, Hovenia dulcis (Rhamnaceae), em bovinos ${ }^{1}$
}

\author{
Thalita C. Cardoso², Tiffany Emmerich ${ }^{2}$, Nathalia S. Wicpolt ${ }^{2}$, Daiane Ogliari², \\ Sandra D. Traverso ${ }^{2}$ e Aldo Gava ${ }^{2 *}$
}

\begin{abstract}
Cardoso T.C., Emmerich T., Wicpolt N.S., Ogliari D., Traverso S.D. \& Gava A. 2015. [Experimental poisoning by the fruits of Hovenia dulcis (Rhamnaceae) in cattle.] Intoxicação experimental pelos frutos de uva-Japão, Hovenia dulcis (Rhamnaceae), em bovinos. Pesquisa Veterinária Brasileira 35(2):115-118. Setor de Patologia Animal, Faculdade de Veterinária, Universidade do Estado de Santa Catarina, Avenida Luis de Camões 2090, Lages, SC 88520-000, Brazil. E-mail: a2ag@cav.udesc

Hovenia dulcis Thunberg (Japan grape) is a deciduous tree native of China and some parts of Japan. Over the last years, the tree has been used for shading in poultry farms of western and middle western Santa Catarina, spreading in the region. Cattle ingest the ripe fruits of $H$. dulcis fallen to the ground. The fruits were suspected to cause poisoning during fall and early winter, period of their maturation. The disease has been reproduced in 2004 by other authors with a single dose of $24.5 \mathrm{~g} / \mathrm{kg}$. In the subsequent years no complaints regarding the toxicity of $H$. dulcis fruits have been reported, although many breeders observed that cattle continued to consume them during the ripening season. We administered the fruits at single doses of $30-50 \mathrm{~g} / \mathrm{kg}$ to nine calves; only two of them became seriously ill and one died. The clinical picture and lesions were similar to the poisoning reproduced in 2004; however, the dose required to reproduce the poisoning was $100 \%$ higher than the lethal dose found in 2004.
\end{abstract}

INDEX TERMS: Poisonous plants, Japan grape, Hovenia dulcis, Rhamnaceae, plant poisoning, cattle.

RESUMO.- Hovenia dulcis Thunberg (uva-Japão) é uma árvore caducifolia nativa da China e de alguns lugares do Japão. Nos últimos anos essa planta foi utilizada como forma de sombreamento para aviários no Oeste e Meio-Oeste de Santa Catarina e passou a disseminar-se por toda a região. Os bovinos comem avidamente seus frutos maduros quando caem ao chão. Suspeitas de intoxicação ocorreram no outono e início de inverno e coincidiam com a maturação dos frutos. A doença foi reproduzida em 2004 por outros autores através da administração dos frutos a bovinos, em dose única de $24,5 \mathrm{~g} / \mathrm{kg}$. Nos anos subsequentes não ocorreram reclamações sobre a intoxicação pelos frutos dessa planta, embora, muitos criadores afirmavam que os bovinos, na temporada de maturação, continuavam a ingerir os frutos. Experimentalmente, nove bovinos que receberam frutos da planta em doses únicas entre 30 e $50 \mathrm{~g} / \mathrm{kg}$, ape-

\footnotetext{
${ }^{1}$ Recebido em 8 de julho de 2014.

Aceito para publicação em 4 de fevereiro de 2015.

${ }^{2}$ Departamento de Patologia Animal, Universidade do Estado de Santa Catarina, Av. Luís de Camões 2090, Lages, SC 88520-000, Brasil. *Autor para correspondência: $\underline{\text { a2ag@cav.udesc.br }}$
}

nas dois bovinos adoeceram gravemente e um morreu. 0 quadro clínico e as lesões foram semelhantes à intoxicação reproduzida em 2004; porém, a dose necessária para reproduzirmos a doença foi $100 \%$ superior à dose tóxica preconizada como letal em 2004.

TERMOS DE INDEXAÇÃO: Plantas tóxicas, uva-Japão, Hovenia dulcis, Rhamnaceae, intoxicação por plantas, bovinos.

\section{INTRODUÇÃO}

Hovenia dulcis é uma árvore pertencente à família Rhamnaceae e popularmente conhecida como "uva-Japão", "tripa-de-galinha", "caju-japonês" e "chico- magro" (Carvalho 1994), é amplamente distribuída em toda região sul do Brasil. Considerada exótica e invasora, pode atingir até 15 metros de altura (Zenni \& Ziller 2011). A floração ocorre na primavera - verão e a frutificação no inverno (Backes \& Irgang 2004). Em Santa Catarina essa planta foi inicialmente utilizada para proteção de aviários. Posteriormente, invadiu campos e matas e atualmente é encontrada na maioria das regiões, principalmente no Oeste do estado. Destaca-se por produzir grande quantidade de frutos, os quais são 
palatáveis e frequentemente ingeridos pelos bovinos. De acordo com informações de produtores rurais e veterinários, a ingestão desses frutos seria a causa de uma enfermidade de bovinos caracterizada por apatia, anorexia, atonia ruminal, tremores musculares, fezes secas, e em pequena quantidade, ou pastosas a líquidas e andar cambaleante, resultando em morte ou recuperação. Há indícios de que esses sinais clínicos seriam mais acentuados em bovinos que ingeriam ração e ou silagem, associado ao consumo dos frutos de "uva-Japão". Na literatura, não há relatos de intoxicação espontânea pelos frutos maduros de $H$. dulcis em bovinos. A intoxicação pelo consumo dos frutos de $H$. dulcis é descrita em caprinos que tiveram acesso aos frutos após a derrubada da árvore e estes foram consumidos durante cinco dias. 0 quadro clínico caracterizava-se por sinais nervosos superagudo ou agudo. A principal lesão macroscópica foi observada no encéfalo, caracterizada por achatamento das circunvoluções da superfície dorsal dos hemisférios. Pela microscopia, os neurônios da substância cinzenta do córtex tinham citoplasma condensado e núcleo picnótico, além de espaços perineuronais e perivasculares proeminentes (Colodel et al. 1998).

Experimentos realizados em bovinos com os frutos de "uva- Japão" produziram alterações clínicas semelhantes às descritas pelos produtores e doses superiores a $24,5 \mathrm{~g} / \mathrm{kg}$ foram letais. Como principais alterações macroscópicas foi observado fígado com aspecto de noz-moscada e o rúmem e retículo tinham a mucosa pálida. Pela microscopia foram observadas necrose e degeneração vacuolar de hepatócitos acompanhadas de congestão moderada. No rúmen havia necrose moderada da mucosa com formação de pequenas vesículas (Gava et al. 2004). A partir do ano de 2005 as informações sobre suspeita de intoxicação por "uva-Japão" foram escassas, embora a quantidade de frutos disponíveis para os animais tenham se mantido. 0 presente trabalho tem como objetivo descrever o quadro clínico patológico e avaliar a possível variação da toxicidade dos frutos de $\mathrm{Ho}$ venia dulcis, bem como, o possível agravamento do quadro clínico quando da ingestão dos frutos dessa planta associada à ingestão de alimentos ricos em carboidratos.

\section{MATERIAL E MÉTODOS}

Levantamentos de históricos foram efetuados com produtores e veterinários nos municípios da região Oeste e Meio-Oeste de Santa Catarina. Para experimentação, foram coletados frutos maduros, colhidos do chão (Fig.1) nos municípios de Concórdia, São José do Cedro, Joaçaba e Erval Velho. Os frutos foram administrados, por via oral, em dose única para nove bovinos. Foram utilizados bovinos machos e fêmeas, todos provenientes de propriedades livres dos frutos de "uva-Japão". Os bovinos foram divididos em dois grupos. Grupo I: os que receberam somente o fruto maduro (Bov. $143,144,150$ e 152) e Grupo II: os que receberam o fruto maduro associado à silagem de milho ad libitum (Bov. 147, 148 ,149, 151 e 153). 0 delineamento do experimento com frutos maduros de Hovenia dulcis está representado no Quadro 1. Todos os bovinos do experimento foram mantidos em baias, alimentados com trevo branco (Trifolium repens) e azevém (Lolium multiflorum), e água foi fornecida ad libitum. Os bovinos foram acompanhados, com avaliações clínicas várias vezes ao dia. Um bovino foi necropsiado e fragmentos das principais vísceras foram coletados para exame

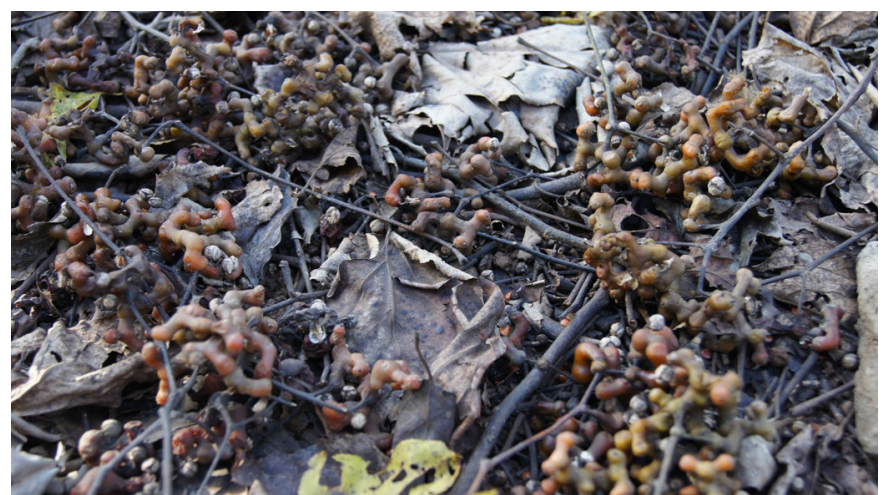

Fig.1. Frutos maduros de "uva-Japão" (Hovenia dulcis) caídos ao chão.

Quadro 1. Delineamento da intoxicação experimental em bovinos com frutos maduros de Hovenia dulcis nos anos de 2011 e 2012

\begin{tabular}{cccccc}
\hline $\begin{array}{c}\text { Bovino } \\
\mathrm{n} \text { o }\end{array}$ & Sexo & Raça & $\begin{array}{c}\text { Peso } \\
(\mathrm{kg})\end{array}$ & $\begin{array}{c}\text { Data da ingestão } \\
\text { da planta }\end{array}$ & $\begin{array}{c}\text { Dose } \\
(\mathrm{g} / \mathrm{kg})\end{array}$ \\
\hline 143 & Macho & Mestiça & 155 & 09.06 .2011 & 30 \\
144 & Macho & Mestiça & 135 & 12.06 .2011 & 40 \\
147 & Macho & Mestiça & 300 & 02.07 .2011 & $30^{*}$ \\
148 & Fêmea & Mestiça & 230 & 02.07 .2011 & $40^{*}$ \\
149 & Fêmea & Holandes & 300 & 05.07 .2011 & $30^{*}$ \\
150 & Macho & Mestiça & 240 & 19.07 .2011 & 40 \\
151 & Macho & Jersey & 210 & 16.05 .2012 & $30^{*}$ \\
152 & Fêmea & Mestiça & 105 & 28.05 .2012 & 40 \\
153 & Fêmea & Mestiça & 150 & 14.06 .2012 & $50^{*}$
\end{tabular}

* Adicionado silagem de milho ad libitum na alimentação.

histológico, fixados em formalina a $10 \%$, processados rotineiramente, corados pela técnica de Hematoxilina-Eosina (HE) e observados no microscópio óptico.

Biópsias hepáticas foram realizadas em oito bovinos, pelo método de punção transtorácica (Braga et al. 1985), fixadas em formol a $10 \%$, processadas rotineiramente e coradas com hematoxilina e eosina (HE). As biópsias foram realizadas entre 24 e 48 horas após a administração dos frutos.

0 Projeto de pesquisa foi aprovado pelo Comitê de Ética de Experimentação Animal (CETEA) da Universidade do Estado de Santa Catarina (CAV/ UDESC) Protocolo nํㅜ 1.07.11.

\section{RESULTADOS}

Dos nove bovinos usados para experimentação, dois adoeceram gravemente (Bov.144 e 153), cinco tiveram manifestações clínicas leves (Bov.143, 147, 148, 151 e 152) e dois não adoeceram (Bov.149 e 150). Os principais sinais clínicos foram anorexia, salivação, tremores musculares, incoordenação motora, cegueira, movimentos frequentes das orelhas e cabeça, atonia ruminal e decúbito esternal. Um bovino (Bov.153), tinha sede intensa e as fezes eram líquidas e fétidas e morreu. 0 início, a gravidade e a evolução dos sinais clínicos são descritos no Quadro 2.

Na necropsia do Bovino 153, havia evidenciação moderada do padrão lobular hepático e leve edema na parede da vesícula biliar. No rúmen, foi encontrada grande quantidade de partes do fruto e sementes misturadas ao conteúdo líquido (Fig.2). Sementes também foram observadas no omaso e abomaso. Nesse último, a mucosa estava avermelhada. No cólon e reto, havia grande quantidade de 
Quadro 2. Evolução e intensidade dos sinais clínicos dos bovinos que receberam experimentalmente frutos maduros e sementes de Hovenia dulcis nos anos de 2011 e 2012

\begin{tabular}{|c|c|c|c|c|c|c|}
\hline \multicolumn{3}{|c|}{ Planta administrada } & \multicolumn{4}{|c|}{ Evolução após a ingestão dos frutos e sementes } \\
\hline $\begin{array}{l}\text { Bovino } \\
\text { № }\end{array}$ & Data & $\begin{array}{c}\text { Dose } \\
\text { (g/ kg) }\end{array}$ & $\begin{array}{l}\text { Intensidade dos } \\
\text { sinais clínicos }\end{array}$ & $\begin{array}{c}\text { Início dos } \\
\text { sinais clínicos }\end{array}$ & Recuperação & Morte \\
\hline 143 & 09.06 .2011 & 30 & + & $17 \mathrm{~h}$ & $24 \mathrm{~h}$ & - \\
\hline 144 & 12.06 .2011 & 40 & +++ & $14 \mathrm{~h}$ & $78 \mathrm{~h}$ & - \\
\hline 147 & 02.07 .2011 & $30 *$ & + & $12 \mathrm{~h}$ & $24 \mathrm{~h}$ & - \\
\hline 148 & 02.07 .2011 & $40^{*}$ & + & $10 \mathrm{~h}$ & $24 \mathrm{~h}$ & - \\
\hline 149 & 05.07 .2011 & $30^{*}$ & - & - & - & - \\
\hline 150 & 19.07.2011 & 40 & - & - & - & - \\
\hline 151 & 16.05 .2012 & $30 *$ & + & $8 \mathrm{~h}$ & $36 \mathrm{~h}$ & - \\
\hline 152 & 28.05 .2012 & 40 & + & $16 \mathrm{~h}$ & $24 \mathrm{~h}$ & - \\
\hline 153 & 14.06 .2012 & $50 *$ & +++ & $14 \mathrm{~h}$ & - & $107 \mathrm{~h}$ \\
\hline
\end{tabular}

Lesão +++ intensa, ++ moderada, + leve, - ausente. *Adicionado silagem de milho ad libitum na alimentação.
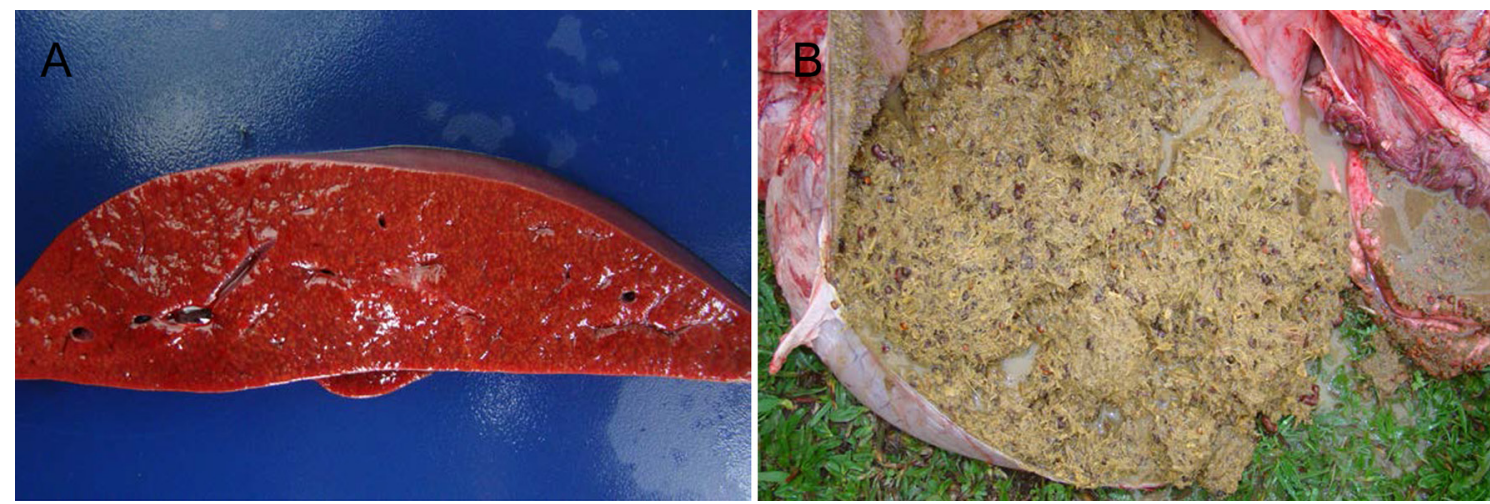

Fig.2. (A) Fígado levemente amarelado com moderada evidenciação do padrão lobular. (B) Rúmen com conteúdo levemente cinza e restos de frutos de Hovenia dulcis.

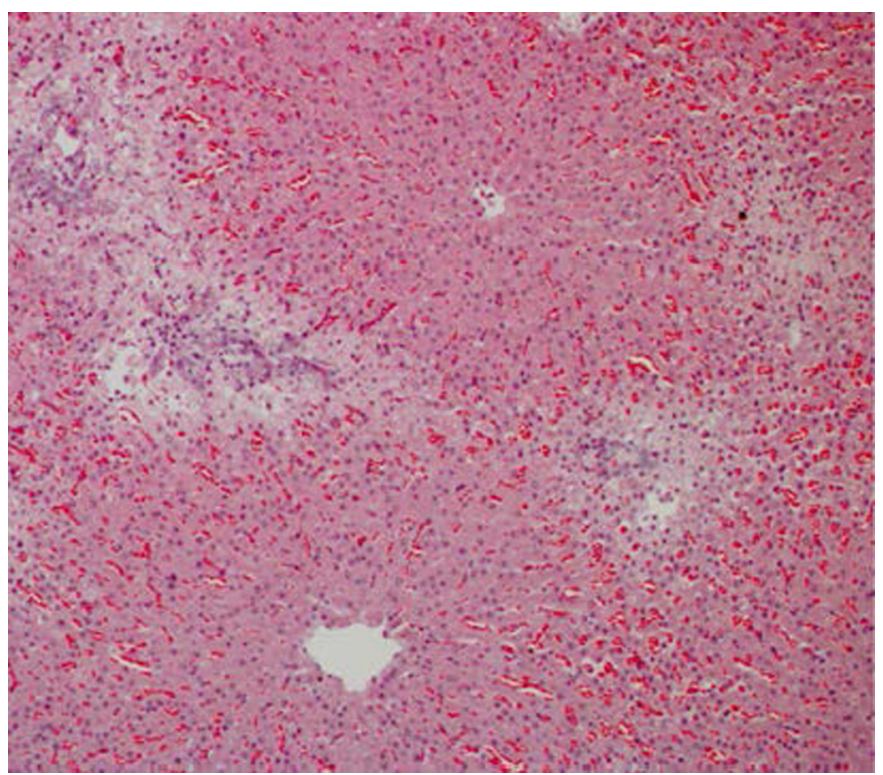

Fig.3. Degeneração vacuolar dos hepatócitos da região periportal do fígado, acompanhada de proliferação do epitélio biliar e congestão dos sinusóides. HE, obj.10x.

muco e a mucosa tinha coloração avermelhada. Através da microscopia observou-se tumefação do citoplasma dos hepatócitos da região perilobular acompanhada por congestão dos sinusóides, proliferação do epitélio biliar, infiltrado de neutrófilos e macrófagos de intensidade leve a mode- rada (Fig.3). Nas biópsias hepáticas não foram observadas lesões.

\section{DISCUSSÃO}

Experimentalmente, a administração dos frutos de Hovenia dulcis em bovinos produziu alterações clínicas e morte em um bovino que recebeu os frutos na dose de $50 \mathrm{~g} / \mathrm{kg}$. Entretanto, doses entre 30 e $40 \mathrm{~g} / \mathrm{kg}$, dos oito bovinos restantes, apenas um, que recebeu $40 \mathrm{~g} / \mathrm{kg}$ apresentou sinais clínicos de forte intensidade e recuperou-se. Nos demais bovinos, os sinais foram leves ou ausentes. Esses resultados sugerem existirem diferenças quanto à resistência individual para com a toxicidade aos frutos de $H$. dulcis. Nos experimentos realizados por Gava et al. (2004), a dose letal para os frutos de "uva-Japão" foi de 24,5g/kg de peso vivo. Também, de acordo com históricos obtidos, a partir de 2005 não foram constatados novos casos de intoxicação pelos frutos dessa planta. Estes fatos sugerem que a toxicidade dos frutos de H. dulcis não é estável.

Experimentalmente os bovinos que adoeceram manifestaram anorexia, fezes pastosas a liquidas, salivação, tremores musculares, incoordenação motora, perda parcial da visão, movimentos frequentes das orelhas e cabeça, atonia ruminal e decúbito esternal. Estes sinais em parte foram semelhantes aos observados por Gava et al. (2004). Os mesmos autores descreveram como principais alterações macroscópicas a coloração vermelha escura e ou amarelada do fígado, o qual tinha evidenciação do padrão lobular. 
A microscopia era caracterizada por necrose massiva dos hepatócitos acompanhada de congestão em um bovino, tumefação dos hepatócitos da região centrolobular e congestão na periferia em outro. 0 bovino do presente estudo, que morreu com dose de $50 \mathrm{~g} / \mathrm{kg}$, apresentou evidenciação moderada dos lóbulos hepáticos e pela microscopia, o citoplasma dos hepatócitos da região periportal era finamente vacuolizado, acompanhada por congestão dos sinusóides, proliferação do epitélio biliar e infiltrado de neutrófilos e macrófagos de intensidade leve a moderada. Dessa forma as lesões macroscópicas e microscópicas encontradas no presente estudo, foram menos graves que as observadas por Gava et al. (2004).

Como os sinais clínicos e lesões macroscópicas e microscópicas em parte são semelhantes às produzidas por plantas de ação hepatotóxica aguda. No diagnóstico diferencial devem ser consideradas as intoxicações por: Dodonea viscosa, encontrada principalmente na região litorânea (Cattani et al. 2004), Cestrum intermedium (Gava et al. 1996, Furlan et al. 2008) encontrada na região Oeste dos três estados do sul, Cestrum corymbosum encontrada no Planalto e Alto Vale do Itajaí (Gava et al. 1991) e Xanthium cavanillesii (Driemeier et al. 1999, Colodel et al. 2000). Também deve ser considerada a intoxicação por larvas de Perreyia flavipes (Soares et al. 2008). Para o diagnóstico diferencial, deve-se levar em consideração que a intoxicação por "uva-Japão" é sazonal, uma vez que os frutos estão disponíveis para os animais apenas no período entre final de maio a julho.

De acordo com informações obtidas de alguns criadores e veterinários, a intoxicação espontânea por $H$. dulcis seria favorecida pela adição, na alimentação dos bovinos, de silagem e/ou ração contendo grãos de cereais. A adição de silagem na alimentação dos bovinos testados parece não contribuir para o agravamento dos sinais clínicos. No Bovino 153 que morreu, não foram visualizadas lesões significativas no rúmem. Entretanto, em um bovino do experimento realizado por Gava et al. (2004), no rúmen foi observado necrose moderada da mucosa com formação de pequenas vesículas e, em alguns segmentos, havia separação entre a mucosa e a submucosa. Desta forma supõe-se que essa lesão pode estar relacionada à ingestão somente dos frutos de H. dulcis, sem a participação da ingestão de carboidratos como a silagem de milho.

\section{CONCLUSÕES}

Pela ausência de suspeitas de intoxicação por Hovenia dulcis nos últimos anos e pelo fato que a dose letal ter sido apenas $50 \mathrm{~g} / \mathrm{kg}$ no presente experimento, conclui-se que os frutos de H. dulcis podem variar em toxicidade.

$\mathrm{O}$ uso de silagem associada à ingestão de frutos de $H$. dulcis não contribuiu para a reprodução da enfermidade.

Agradecimentos.- À Universidade do Estado de Santa Catarina pelo apoio financeiro ao projeto. À CAPES, pela concessão de bolsa de mestrado.

\section{REFERÊNCIAS}

Backes P. \& Irgang B. 2004. Árvores cultivadas no sul do brasil. Guia de Identificação e interesse paisagístico das principais espécies exóticas. Paisagens do Sul, Porto Alegre. 204p.

Braga M.M., Castilhos L.M.L. \& Santos M.N. 1985. Biópsia hepática em bovinos: proposta de nova técnica. Revta Centro Ciênc. Rurais 15(1):79-88.

Carvalho P.E.R. 1994. Ecologia, silvicultura e usos da Uva-do-Japão (Hovenia dulcis Thunberg). Circ. Téc. 23, Embrapa-CNPF, Colombo. 24p.

Cattani C.S.O., Colodel E.M., Traverso S.D., Correa A.M.R. \& Driemeier D. 2004. Intoxicação experimental por Dodonea viscosa em bovinos. Pesq. Vet. Bras. 24:31-34

Colodel E.M., Loretti A.P., Cruz F.C.E. \& Driemeier D. 1998. Polioencefalomalacia em caprinos associada à ingestão dos frutos de Hovenia dulcis (uva-do-Japão). Bolm 25 do Laboratório Regional de Diagnóstico, Pelotas, p.35-42.

Colodel E.M., Driemeier D. \& Pilati C. 2000. Intoxicação experimental pelos frutos de Xanthium cavanillesii (Asteraceae) em bovinos. Pesq. Vet. Bras. 20(1):31-38.

Driemeier D., Irigoyen L.F., Loretti A.P., Colodel E.M. \& Barros C.S.L. 1999. Intoxicação espontânea pelos frutos de Xanthium cavanillesii (Asteraceae) em bovinos no Rio Grande do Sul. Pesq. Vet. Bras. 19(1):1-13.

Furlan F.H., Lucioli J., Borelli V., Junior O.O.F., Rebelatto S.V., Gava A. \& Traverso S.D. 2008. Intoxicação por Cestrum intermedium (Solanaceae) em bovinos no Estado de Santa Catarina. Acta Scient. Vet. 36(3):281-284.

Gava A., Stolf L., Pilati C., Neves D.S. \& Vigano L. 1991. Intoxicação por Cestrum corymbosum var. hirsutum (Solanaceae) em bovinos no Estado de Santa Catarina. Pesq. Vet. Bras. 11(3/4):71-74.

Gava A., Stolf L., Varaschin M.S., Neves D.S., Tigre A.P. \& Lessmann F. 1996. Intoxicação por Cestrum intermedium (Solanaceae) em bovinos. Pesq. Vet. Bras. 16(4):117-120

Gava A., Balbinotti V., Neve D.S., Rocha T.S., Mazaroba S. \& Gava D. 2004. Intoxicação por Hovenia dulcis (uva-do-Japão) em bovinos. Pesq. Vet. Bras. 24(Supl.):25-26.

Soares M.P., Quevedo P.S. \& Schild A.L. 2008. Intoxicação por larvas de Perreyia flavipes em bovinos na região sul do Rio Grande do Sul. Pesq. Vet. Bras. 28(3):169-173.

Zenni R.D. \& Ziller S.R. 2011. An overview of invasive plants in Brazil. Revta Bras. Bot. 34(3):431-446. 